\title{
Synthesis and evaluation for anticonvulsant activity of some N-(5-(substituted)-1,3,4- thiadiazol-2-yl)-2-((5-(substituted)-4H-1,2,4-triazol-3-yl)-amino) acetamide derivatives
}

\author{
Dinesh D. Rishipathak ${ }^{1, *}$, Luketa R. Alai ${ }^{2}$, Pavan B. Udavant ${ }^{3}$ \\ ${ }^{1,3}$ Associate Professor, ${ }^{2}$ PG Scholar, ${ }^{1,2}$ Dept. of Pharmaceutical Chemistry, ${ }^{3}$ Dept. of Pharmacology, MET's Institute of Pharmacy, \\ Bhujbal Knowledge City Adgaon, Nashik, Maharashtra, India \\ *Corresponding Author: \\ Email: drishipathak@gmail.com
}

\begin{abstract}
Several new promising bioactive derivatives of N-(5-(Substituted)-1, 3, 4-thiadiazol-2-yl)-2-((5-(substitutes)-4H-1, 2, 4-triazol-3-yl) amino) acetamide were synthesized. The compounds were obtained in excellent yields. The synthesized compounds were confirmed on the basis of IR and NMR. Acute toxicity study was done to determine the LD 50 of the newly synthesized compounds. Some of the synthesized compounds were evaluated for their anticonvulsant effect by PTZ induced convulsions method. Statistical testing was done by one way ANOVA followed by Dunnett's test. The compounds D-III showed the highest percentage of protection as compared to PTZ, i.e. $80 \%$ at the dose of $20 \mathrm{mg} / \mathrm{kg}$ among the evaluated compounds compared to control.
\end{abstract}

Keywords: 1, 3, 4-thiadiazole, 1, 2, 4-triazole, Anticonvulsant.

\section{Introduction}

Epilepsy is not a diseases, but a syndrome of different cerebral disorders of the Central Nervous System (CNS), which is characterized by paroxysmal, excessive and hypersynchronous discharges of large numbers of neurons. Epilepsy is one of the most common serious neurological disorders characterized by recurrent seizures. ${ }^{1}$

Several newer antiepileptic drugs (such as pregabalin, stiripentol, lamotrigine, levetiracetam, Topiramate) are greatly compromised by severe side effects such as vertigo, ataxia, headache, hirutism, hepatotoxicity, gastrointestinal and cardiovascular. Moreover about 30\% of patients have uncontrolled seizures. The insufficient information on the cellular mechanism of epilepsy in humans and the complex mechanism of action of most of the antiepileptic drugs makes it difficult to use rational methodologies in the field of drug discovery. There is substantial need for the development of new, more effective and less toxic antiepileptic drugs. ${ }^{2}$

The biological activity of compounds mainly depends on their molecular structure. Heterocyclic moieties can be found in a large number of compounds which display large number of biological activity. Thiadiazole is a versatile moiety that exhibits a wide variety of activity due to the presence of $\mathrm{N}=\mathrm{C}-\mathrm{S}$ moiety in the ring. They have become an important class of heterocycles of great interest of researches because of their broad types of biological activity. ${ }^{3}$ thiadiazole composed of two electron-deficient carbon atoms these are interconnected with nitrogen atoms, and a sulfur atom with lone electron pairs with the general formula of $\mathrm{C} 2 \mathrm{H} 2 \mathrm{~N} 2 \mathrm{~S}$. They occur in four isomeric form namely 1,2,3-thiadiazole, 1,2,4-thiadiazole, 1,2,5thiadiazole, 1,3,4-thiadiazole. Among them 1,3,4thiadiazole ring exhibits more versatile activities.

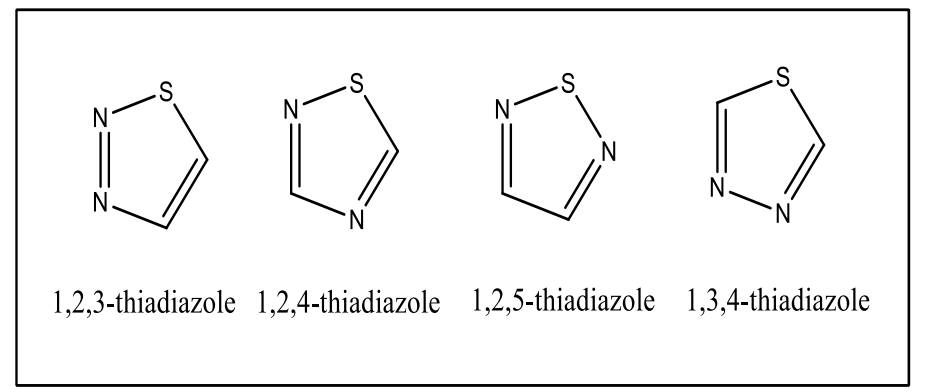

Fig. 1: Isomers of thiadiazole

1,3,4-thiadiazole have wide range of pharmacological activities such as antibacterial, antifungal, antitubercular, antihepatatitis B viral, antileishmanial, anti-inflammatory, analgesic, antimicrobial, antitubercular, and anticonvulsant activities. These important biological activities encouraged researches to find out different methods for synthesis of new thiadiazoles using different synthons, such as thiosemicarbazides, thiocarbazides, dithiocarbazates, thioacylhydrazines, acylhydrazines, and bithiourea. $^{4}$ 
Triazole is a heterogeneous five-membered ring compound containing three nitrogen atoms and two carbon atoms with molecular formula $\mathrm{C}_{2} \mathrm{H}_{3} \mathrm{~N}_{3}$. There are two type of isomers that vary in the relative positions of the nitrogen. Each of isomers have two tautamer's that vary by which nitrogen bonded with hydrogen. Triazole exists as two isomers 1,2,3-triazoles and 1,2,4-triazoles. ${ }^{5}$ out of the two triazoles,1,2,4-triazole have drawn great attention to medicinal chemist from two decades due to its wide variety of activity, low toxicity and good pharmacokinetic and Pharmacodynamic profiles. ${ }^{6}$

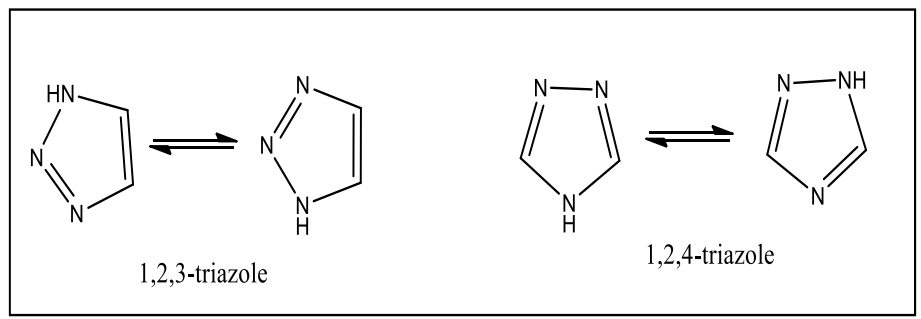

Fig. 2: Triazole Isomers

1,2,4-triazole and its derivatives posses widely differing activities e.g. antibacterial, antifungal, anticancer, antitubercular, anti-inflammatory, analgesic, antiviral, anticonvulsant, anticorrosive, antiigrane, sedative, diuretic and anti-HIV etc. ${ }^{7}$

\section{Materials and Method}

All the chemicals were of laboratory reagent grade and were obtained from LobaChemie, Sigmaldrich and SDFCl. Melting point were taken in one end sealed glass capillary using liquid paraffin in Thiele's tube and were corrected. Analytical thin-layer chromatography was performed on 60F254 precoated silica gel plates (Merck) to establish identity of reactants and products monitored in between reactions as well at the end for completion of reaction. The spots were visualized in UV chamber or by iodine vapors in an enclosed chamber. The solvent system used for Thin-layer Chromatography was Ethyl acetate: n- hexane (7:3) and Benzene: methanol (7:3). Infra-Red spectra of compounds were recorded on 1) Perkin Elmer Spectrum Two FT-IR spectrometer in the range of 4000$200 \mathrm{~cm}^{-1}$, Pharmaceutical Analysis. 2) DRS on a Shimadzu 1000 FTIR spectrometer in the range of 4000$200 \mathrm{~cm}^{-1}$, Chemistry Department KTHM College Nasik. Proton $(1 \mathrm{H})$ Nuclear Magnetic Resonance spectra of compounds were recorded on Bruker Avance II 400 NMR Spectrophotometer using DMSO solvent, at SAIF, Punjab University, Chandigarh. All microwave reactions were carried on 'Catalyst System' CATA 2R- Scientific Microwave Synthesizer with power setting from P-1 and $\mathrm{H}(700 \mathrm{~W})$. The completion of the reaction was monitored by TLC.

\section{Synthetic Scheme}

Step 1: Procedure for Synthesis of 2-amino, 5-substituted phenyl, 1,3,4-thiadiazole. ${ }^{8}$

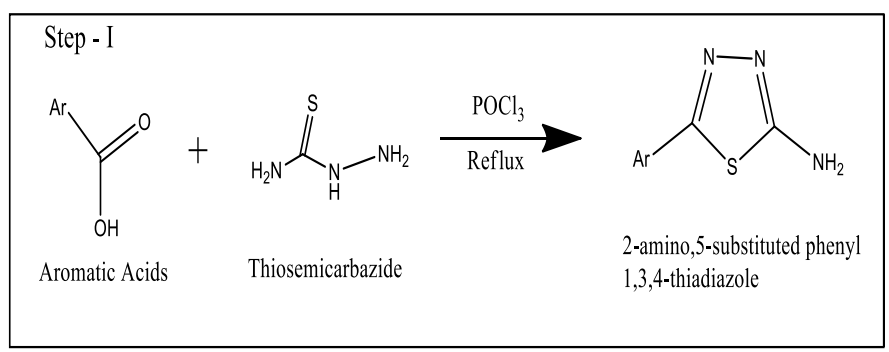

Phosphorous oxychloride (0.034 mole) was added drop-wise to an ice cold mixture of thiosemicarbazide powder (0.01 mole) and aromatic acid (0.01 mole) with stirring, reflux was continued for the hours mentioned in table no.1. Mixture was cooled to room temperature, added to $250 \mathrm{ml}$ stirred ice cold water. Neutralized with $10 \%$ sodium carbonate solution. The precipitate was filtered, washed with water and recrystallized from aqueous ethanol. The completion of reaction was checked by TLC using solvent system (Ethyl acetate: $n$-hexane).

Table 1: Reflux time required for completion of reaction

\begin{tabular}{|c|c|l|c|}
\hline Sr. No. & Code & \multicolumn{1}{|c|}{ Ar } & Time (Hours) \\
\hline 1 & a & p- $\mathrm{NO}_{2}-\mathrm{C}_{6} \mathrm{H}_{4-}$ & 1.5 \\
\hline 2 & b & o- $-\mathrm{I}_{6} \mathrm{H}_{4} 4^{-}$ & 3 \\
\hline 3 & c & o-Cl- $\mathrm{C}_{6} \mathrm{H}_{4-}$ & 2.5 \\
\hline 4 & d & p-Cl- $\mathrm{C}_{6} \mathrm{H}_{4-}$ & 1 \\
\hline
\end{tabular}




\begin{tabular}{|l|l|l|l|}
\hline 5 & e & 2-pyridyl & 3 \\
\hline 6 & f & o-OH- $\mathrm{C}_{6} \mathrm{H}_{4^{-}}$ & 2 \\
\hline
\end{tabular}

Step 2: Procedure for condensation of 2-Chloroacetyl amino, 5-substituted phenyl, 1,3,4-thiadiazole.(Microwave)

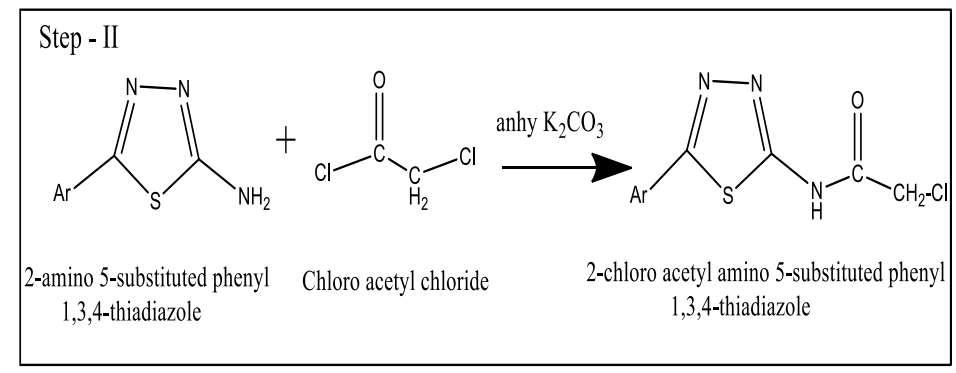

Chloro acetyl chloride (0.01 mole) was added drop-wise to 2-amino 5- substituted 1,3,4-thiadiazole ( 0.01 mole) in $\mathrm{DMF}$ at cold condition in presence of anhydrous potassium carbonate $(0.01$ mole). This mixture was irradiated under microwave at power and time mentioned in table 2. The resulting mixture was allowed to cool and crushed ice was added and stirred, precipitate was collected and dried. Purity of product was checked by using TLC using solvent system (Ethyl acetate: n-hexane 7:3).

Table 2: Reaction condition for synthesis of 2-Chloroacetyl amino, 5 -substituted phenyl, 1,3,4-thiadiazole.

\begin{tabular}{|c|c|l|c|c|}
\hline Sr. No. & Code & \multicolumn{1}{|c|}{$\mathbf{A r}$} & Time (Min) & Power (Watt) \\
\hline 1 & $\mathrm{~A}$ & $\mathrm{p}-\mathrm{NO}_{2}-\mathrm{C}_{6} \mathrm{H}_{4}-$ & 40 & 350 \\
\hline 2 & $\mathrm{~B}$ & $\mathrm{o}-\mathrm{I}-\mathrm{C}_{6} \mathrm{H}_{4^{-}}$ & 15 & 350 \\
\hline 3 & $\mathrm{C}$ & $\mathrm{o}-\mathrm{Cl}-\mathrm{C}_{6} \mathrm{H}_{4-}$ & 30 & 420 \\
\hline 4 & $\mathrm{D}$ & $\mathrm{p}-\mathrm{Cl}-\mathrm{C}_{6} \mathrm{H}_{4-}$ & 20 & 350 \\
\hline 5 & E & 2-pyridyl & 15 & 350 \\
\hline
\end{tabular}

Step 3: Procedure for Synthesis of 3-amino 5-substituted phenyl 1,2,4-triazole. ${ }^{9}$

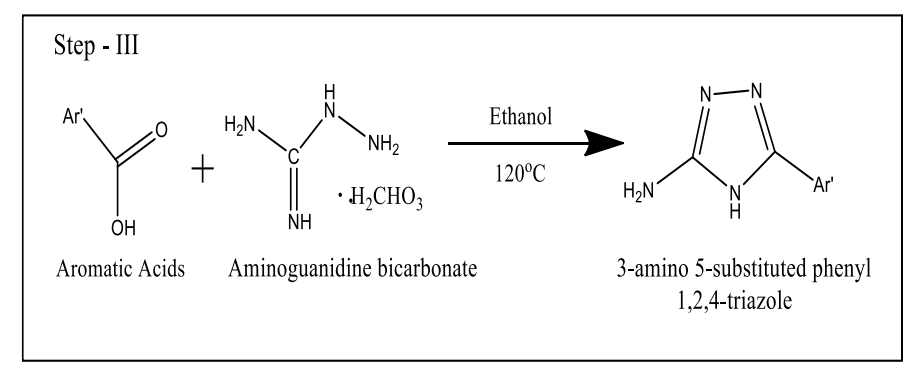

A mixture of Aminoguanidine bicarbonate $(0.01$ mole) and Aromatic acids $(0.01$ mole $)$ in ethanol was warmed manually, till the bubbles of carbon dioxide was evolved. The mixture was irradiated under microwave at power and time mentioned in table no.3. The solution was evaporated to collect the solid. The solid was recrystallized with ethanol. Purity of product was checked by TLC using solvent system (Benzene: Methanol 7:3).

Table 3: Reaction condition for synthesis of 3-amino 5-substituted phenyl 1,2,4-triazole.

\begin{tabular}{|c|c|l|c|c|}
\hline Sr. No. & Code & Ar' & Time (Min) & Power (Watt) \\
\hline 1 & I & p- $\mathrm{NO}_{2}-\mathrm{C}_{6} \mathrm{H}_{4-}$ & 30 & 420 \\
\hline 2 & II & p-Cl- $\mathrm{C}_{6} \mathrm{H}_{4^{-}}$ & 40 & 420 \\
\hline 3 & II & $\mathrm{C}_{6} \mathrm{H}_{4}-$ & 30 & 350 \\
\hline 4 & IV & H- & 15 & 350 \\
\hline
\end{tabular}

Step 4: Procedure for condensation of N-(5-(Substituted)-1,3,4-thiadiazol-2-yl)-2-((5-(substituted)-4H-1,2,4-triazol-3yl)amino)acetamide. 


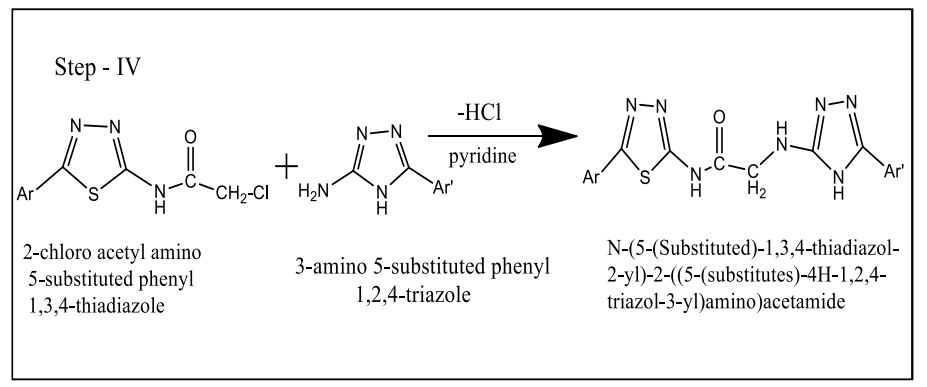

A mixture of 2-Chloroacetyl amino, 5-substituted phenyl, 1,3,4-thiadiazole( $0.001 \mathrm{~mole})$ and 3-amino 5-substituted phenyl 1,2,4-triazole $(0.001$ mole) using solvent DMF $(15 \mathrm{ml})$ in presence of pyridine, was heated manually for time mentioned in table no.4. The mixture was allowed to cool and crushed ice was added. The precipitate was filtered and solid was dried and recrystallized from ethanol. Purity of product was checked by TLC using solvent system (Ethyl acetate: n-hexane 7:3).

Table 4: Substituents of N-(5-(Substituted)-1,3,4-thiadiazol-2-yl)-2-((5-(substitutes)-4H-1,2,4-triazol-3yl)amino)acetamide time required for completion of reactions

\begin{tabular}{|c|c|c|c|c|}
\hline S. No. & Code & Ar & Ar' & Time (Min) \\
\hline 1 & A-II & $\mathrm{p}-\mathrm{NO}_{2}-\mathrm{C}_{6} \mathrm{H}_{4^{-}}$ & $\mathrm{p}-\mathrm{Cl}-\mathrm{C}_{6} \mathrm{H}_{4}-$ & 40 \\
\hline 2 & A-III & $\mathrm{p}-\mathrm{NO}_{2}-\mathrm{C}_{6} \mathrm{H}_{4}^{-}$ & $\mathrm{C}_{6} \mathrm{H}_{4^{-}}$ & 35 \\
\hline 3 & B-I & o-I- $\mathrm{C}_{6} \mathrm{H}_{4^{-}}$ & $\mathrm{p}-\mathrm{NO}_{2}-\mathrm{C}_{6} \mathrm{H}_{4-}$ & 45 \\
\hline 4 & B-II & o-I- $\mathrm{C}_{6} \mathrm{H}_{4}-$ & p-Cl- $\mathrm{C}_{6} \mathrm{H}_{4^{-}}$ & 55 \\
\hline 5 & C-II & o-Cl- $\mathrm{C}_{6} \mathrm{H}_{4-}$ & $\mathrm{p}-\mathrm{Cl}-\mathrm{C}_{6} \mathrm{H}_{4-}$ & 40 \\
\hline 6 & D-I & p-Cl- $\mathrm{C}_{6} \mathrm{H}_{4-}$ & $\mathrm{p}-\mathrm{NO}_{2}-\mathrm{C}_{6} \mathrm{H}_{4}-$ & 50 \\
\hline 7 & D-II & p-Cl- $\mathrm{C}_{6} \mathrm{H}_{4-}$ & $\mathrm{p}-\mathrm{Cl}-\mathrm{C}_{6} \mathrm{H}_{4-}$ & 35 \\
\hline 8 & D-III & p-Cl- $\mathrm{C}_{6} \mathrm{H}_{4-}$ & $\mathrm{C}_{6} \mathrm{H}_{4^{-}}$ & 45 \\
\hline 9 & D-IV & $\mathrm{p}-\mathrm{Cl}-\mathrm{C}_{6} \mathrm{H}_{4-}$ & H- & 55 \\
\hline
\end{tabular}

Pharmacological evaluation: All the experiments were conducted according to the guidelines of Committee for Purpose of Control and Supervision of Experiments on Animals (CPCSEA), Ministry of Environments and Forests, Government of India with their procedures and protocols reviewed and approved by the Institutional Animal Ethical Committee (IAEC), constituted under CPCSEA. Swiss Albino male mice, weighing 22-30gm were used for the study. The animals were purchased from Hafkine's Institute, at Parel, Mumbai, India. Animals were housed in registered animal house of MET's Institute of Pharmacy, Nashik (Registration no.1344/ac/10/CPCSEA). Ambient temperature of $25 \pm 1$ ${ }^{\circ} \mathrm{C}$, relative humidity of $45-55 \%$ and $12 \mathrm{hrs}$ light: $12 \mathrm{hrs}$ dark cycles were maintained in the animal house. The animals had free access to water and standard pelleted diet except during experimentation food and water was withheld.

Acute toxicity studies: OECD guidelines (no.425) were followed for acute toxicity studies. Acute intraperitoneal (i.p.) toxicity test was carried out for determining median Lethal Dose (LD50). Animals were dosed two at a time at a minimum of 48 hours intervals. Doses were selected from the sequence $2000,550,175,55,17.5,5.5,1.75$ $\mathrm{mg} / \mathrm{kg}$ with 5 animals per group. Each animal was observed carefully for the signs of toxicity as well as for mortality in the first 30 minutes after dosing and then occasionally for further 4 hours and daily thereafter for a period of 14 days. The number of mice dying during 48 hours period was recorded.

Anticonvulsant activity (PTZ induced convulsion method): The drugs were freshly prepared. Pentylenetetrazole (Dose: $80 \mathrm{mg} / \mathrm{kg}$, i.p.), a stock solution containing $20 \mathrm{mg} / 5 \mathrm{~mL}$ was prepared by dissolving it in distilled water. Diazepam (Dose: $2 \mathrm{mg} / \mathrm{kg}$, i.p.), a stock solution containing $0.2 \mathrm{mg} / \mathrm{mL}$ was prepared by dissolving it in distilled water. All the test compounds were insoluble in water hence they were dissolved in DMSO. The doses of test compounds were $10 \mathrm{mg} / \mathrm{kg}$, i.p. and $20 \mathrm{mg} / \mathrm{kg}$, i.p., a stock solution containing $10 \mathrm{mg} / 5 \mathrm{ml}$ was prepared. The injection volume was $1 \mathrm{~mL} / 100 \mathrm{gm}$ of body weight of animal.

Group of five mice were used. One group was used for studying the effects of Pentylenetetrazole alone (Control) and the other one for studying the protective effects of Diazepam (Standard). PTZ ( $80 \mathrm{mg} / \mathrm{kg}$, i.p.) was administered half an hour after the administration of Diazepam and the test compounds. The test group animals were observed for onset of convulsions, number of convulsions and percentage of protection. ${ }^{10}$

\section{Results}

Physicochemical properties and spectral analysis of 2amino, 5-substituted phenyl, 1,3,4-thiadiazole.

2-amino-5-(4-nitro phenyl)-1,3,4-thiadiazole (a). 
Mol.Formula- $\mathrm{C}_{8} \mathrm{H}_{6} \mathrm{~N}_{4} \mathrm{O}_{2} \mathrm{~S}$ Mol.wt- 222.22 M.P.-256$260^{\circ} \mathrm{C}$ Yield \%- 92; IR ( $\left.\mathrm{KBr}\right) \mathrm{cm}^{-1}-\mathrm{NH} 2$ (primary amine) 3423.34, 3103.16; C=N 1454.96; C-S 687.96; C-H (Aromatic) 2980.9; -NO2 1504.51, 1379.82.

2-amino-5-(2-iodo phenyl)-1,3,4-thiadiazole (b).

Mol.Formula- $\mathrm{C}_{8} \mathrm{H}_{6} \mathrm{IN}_{3} \mathrm{~S}$ Mol.wt- 302.93 M.P.-190$194^{\circ} \mathrm{C}$ Yield \%- 67; IR (KBr) cm ${ }^{-1}-\mathrm{NH} 2$ (primary amine) 3420, 3200; C=N 1513.91; C-S 687.96; C-H (Aromatic) 2977.24.

2-amino-5-(2-choloro phenyl)-1,3,4-thiadiazole (c). Mol.Formula- $\mathrm{C}_{8} \mathrm{H}_{6} \mathrm{ClN}_{3} \mathrm{~S}$ Mol.wt- 211.67 M.P.-216$218^{\circ} \mathrm{C}$ Yield \%- 72; IR (KBr) cm ${ }^{-1}-\mathrm{NH} 2$ (primary amine) 3277.93, 3104.87; C=N 1509.09; C-S 657.79, C-H (Aromatic) 2978.62; -Cl 715.52.

2-amino-5-(4-choloro phenyl)-1,3,4-thiadiazole (d). Mol.Formula- $\mathrm{C}_{8} \mathrm{H}_{6} \mathrm{ClN}_{3} \mathrm{~S}$ Mol.wt- 211.67 M.P.-230$234^{\circ} \mathrm{C}$ Yield \%- 89; IR (KBr) cm ${ }^{-1}-\mathrm{NH} 2$ (primary amine) 3277.93, 3104.87; $\mathrm{C}=\mathrm{N}$ 1554.77; C-S 680.94; C-H (Aromatic) 3171.04; -Cl 739.13.

Physicochemical properties and spectral analysis of 3amino 5-substituted phenyl 1,2,4-triazole

3- amino-5-(4-nitro phenyl)-1,2,4-triazole (I)

Mol.Formula- $\mathrm{C}_{8} \mathrm{H}_{6} \mathrm{~N}_{4} \mathrm{O}_{2} \mathrm{~S}$ Mol.wt- 205.17 M.P.-190$194^{\circ} \mathrm{C} \%$ Yield- 89; IR (KBr) cm ${ }^{-1}-\mathrm{NH} 2$ (primary amine) 3498.87, 3344.57; C=N 1562.34; N-H 3043.67; C-N 1334.74; C-H (Aromatic) 2850.79; -NO2 1508.33, 1388.75 .

3- amino-5-(4-chloro phenyl)-1,2,4-triazole (II).

Mol.Formula- $\mathrm{C}_{8} \mathrm{H}_{6} \mathrm{~N}_{4} \mathrm{O}_{2} \mathrm{~S}$ Mol.wt- 194.62 M.P.-130$132^{\circ} \mathrm{C} \%$ Yield- 82; IR (KBr) cm ${ }^{-1}-\mathrm{NH} 2$ (primary amine) 3448.72, 3344.57; C=N 1543.05; N-H 3035.96; C-N 1381.03; C-H (Aromatic) 2870.08; -Cl 763.81.

\section{3- amino-5-phenyl-1,2,4-triazole (III).}

Mol.Formula- $\mathrm{C}_{8} \mathrm{H}_{6} \mathrm{~N}_{4} \mathrm{O}_{2} \mathrm{~S}$ Mol.wt- 160.18 M.P.-150$154^{\circ} \mathrm{C} \%$ Yield- 77; IR (KBr) cm ${ }^{-1}-\mathrm{NH} 2$ (primary amine) $3406.29,3300 ; \quad \mathrm{C}=\mathrm{N}$ 1543.05; N-H 3066.82; C-N 1384.89; C-H (Aromatic) 2785.21.

3- amino-5H-1,2,4-triazole (IV).

Mol.Formula- $\mathrm{C}_{8} \mathrm{H}_{6} \mathrm{~N}_{4} \mathrm{O}_{2} \mathrm{~S}$ Mol.wt- 84.08 M.P.-250$254^{\circ} \mathrm{C} \%$ Yield- 90; IR (KBr) cm ${ }^{-1}-\mathrm{NH} 2$ (primary amine) 3406.29, 3321.42; C=N 1546.91; N-H 3197.98; C-N 1377.17.

Physicochemical properties and spectral analysis of n(5-(substituted)-1,3,4-thiadiazol-2-yl)-2-((5(substituted)-4h-1,2,4-triazol-3-yl)amino)acetamide. 2-((5-(4-chlorophenyl)-4H-1,2,4-triazol-3-yl)amino)$\mathrm{N}$-(5-(4-nitrophenyl)-1,3,4-thiadiazol-2-yl)acetamide (A-II).

Mol.Formula- $\mathrm{C}_{8} \mathrm{H}_{6} \mathrm{IN}_{3} \mathrm{~S}$ Mol.wt- 913.73 M.P.-258$260^{\circ} \mathrm{C}$ Yield \%- 91; IR (KBr) cm ${ }^{-1}-\mathrm{NH} 2$ (primary amine) $3400,3325.28 ; \mathrm{C}=\mathrm{O}$ 1591.27; $\mathrm{C}=\mathrm{N}$ 1546.91; N-H 2920.23; C-N 1342.46; C-S 615.29; C-H (Aromatic) 2850.79; -NO2 1514.12, 1390.68; -Cl 773.46. $1 \mathrm{H}$ NMR (400MHZ DMSO) $\delta$ ppm-8.050 (aromatic), 8.29 (aromatic), 8.76 (sec. amide) 3.94 (-CH2), 4.85 (aromatic C-NH).

N-(5-(4-nitrophenyl)-1,3,4-thiadiazol-2-yl)-2-((5phenyl-4H-1,2,4-triazol-3-yl)amino) acetamide(A-III).
Mol.Formula- $\mathrm{C}_{8} \mathrm{H}_{6} \mathrm{IN}_{3} \mathrm{~S}$ Mol.wt- 422.42 M.P.-246$250^{\circ} \mathrm{C}$ Yield \%- 65; IR (KBr) cm $\mathrm{cm}^{-1}-\mathrm{NH} 2$ (primary amine) $3300, \quad 3298.28 ; \quad \mathrm{C}=\mathrm{O} 1597.06 ; \quad \mathrm{C}=\mathrm{N} \quad 1554.63 ; \quad \mathrm{N}-\mathrm{H}$ 3111.18; C-N 1342.46; C-S 610; C-H (Aromatic) 2922.16; -NO2 1516.05, 1390.68.

N-(5-(4-nitrophenyl)-1,3,4-thiadiazol-2-yl)-2-((5phenyl-4H-1,2,4-triazol-3-yl) amino) acetamide (B-I). Mol.Formula- $\mathrm{C}_{8} \mathrm{H}_{6} \mathrm{IN}_{3} \mathrm{~S}$ Mol.wt- 548.32 M.P.-130$132^{\circ} \mathrm{C}$ Yield \%- 60; IR (KBr) cm $\mathrm{cm}^{-1}-\mathrm{NH} 2$ (primary amine) 3307.92; $\mathrm{C}=\mathrm{O} 1571.99 ; \mathrm{C}=\mathrm{N} 1554.63 ; \mathrm{N}-\mathrm{H}$ 2922.16; CS 610; C-H (Aromatic) 2800; -NO2 1521.84, 1390.68; -I 509.21 .

2-((5-(4-chlorophenyl)-4H-1,2,4-triazol-3-yl)amino)$\mathrm{N}$-(5-(2-iodophenyl)-1,3,4-thiadiazol-2-yl)acetamide. (B-II)

Mol.Formula- $\mathrm{C}_{8} \mathrm{H}_{6} \mathrm{IN}_{3} \mathrm{~S}$ Mol.wt- 1075.53 M.P.-280$284^{\circ} \mathrm{C}$ Yield \%- 89; $\mathrm{IR}(\mathrm{KBr}) \mathrm{cm}^{-1}-\mathrm{NH} 2$ (primary amine) 3331.07; C=O 1591.27; C=N 1546.68; N-H 3111.18; CN 1390.68; C-S 686.66; C-H (Aromatic) 2922.16; -Cl 775.38; -I 524.64. 1H NMR (400MHZ DMSO) $\delta$ ppm 7.2613 (aromatic), 7.2658 (aromatic), 7.2776 (aromatic), 7.2823 (aromatic), 8.6 (sec. amide), 2.51 (CH2), 3.37 (aromatic C-NH).

N-(5-(2-chlorophenyl)-1,3,4-thiadiazol-2-yl)-2-((5-(4chlorophenyl)-4H-1,2,4-triazol-3-yl) amino) acetamide (C-II).

Mol.Formula- $\mathrm{C}_{8} \mathrm{H}_{6} \mathrm{IN}_{3} \mathrm{~S}$ Mol.wt- 892.63 M.P.-148$150^{\circ} \mathrm{C}$ Yield \%- 83; $\mathrm{IR}(\mathrm{KBr}) \mathrm{cm}^{-1}-\mathrm{NH} 2$ (primary amine) 3298.28, 3157.47; $\mathrm{C}=\mathrm{O}$ 1589.34; $\mathrm{C}=\mathrm{N}$ 1535.34; N-H 3100; C-N 1300.02; C-S 613.36; C-H (Aromatic) 2920.23; -Cl 761.88. 1H NMR (400MHZ DMSO) $\delta \mathrm{ppm}$ 7.7452 (aromatic), 7.4585 (aromatic), 7.4747 (aromatic), 7.5509 (aromatic), 8.6875 (sec. amide), 3.6872 (-CH2), 4.2012 (aromatic C-NH).

N-(5-(4-chlorophenyl)-1,3,4-thiadiazol-2-yl)-2-((5-(4nitrophenyl)-4H-1,2,4-triazol-3-yl) amino) acetamide (D-I).

Mol.Formula- $\mathrm{C}_{8} \mathrm{H}_{6} \mathrm{IN}_{3} \mathrm{~S}$ Mol.wt- 456.87 M.P.-260$262^{\circ} \mathrm{C}$ Yield \%- 74; IR ( $\left.\mathrm{KBr}\right) \mathrm{cm}^{-1}-\mathrm{NH} 2$ (primary amine) 3298.28, 3149.76; $\mathrm{C}=\mathrm{O}$ 1593.20; $\mathrm{C}=\mathrm{N} 1431.18 ; \mathrm{N}-\mathrm{H}$ 2920.23; C-N 1342.46; C-S 651.94; C-H (Aromatic) 2850.79; -NO2 1535.34, 1303.88; -Cl 761.88.

N-(5-(4-chlorophenyl)-1,3,4-thiadiazol-2-yl)-2-((5-(4chlorophenyl)-4H-1,2,4-triazol-3-yl) amino)acetamide(D-II).

Mol.Formula- $\mathrm{C}_{8} \mathrm{H}_{6} \mathrm{IN}_{3} \mathrm{~S}$ Mol.wt- 446.31 M.P.-200$204^{\circ} \mathrm{C}$ Yield \%- 69; IR (KBr) cm ${ }^{-1}-\mathrm{NH} 2$ (primary amine) 3298.28, 3153.6; $\mathrm{C}=\mathrm{O}$ 1591.27; $\mathrm{C}=\mathrm{N} 1431.18 ; \mathrm{N}-\mathrm{H}$ 2920.23; C-N 1301.95; C-S 651.94; C-H(Aromatic) 2850.79; -Cl 761.88.

N-(5-(4-chlorophenyl)-1,3,4-thiadiazol-2-yl)-2-((5phenyl-4H-1,2,4-triazol-3-yl) amino) acetamide (DIII).

Mol.Formula- $\mathrm{C}_{8} \mathrm{H}_{6} \mathrm{IN}_{3} \mathrm{~S}$ Mol.wt- 411.87 M.P.-176$180^{\circ} \mathrm{C}$ Yield \%- 88; IR (KBr) cm ${ }^{-1}-\mathrm{NH} 2$ (primary amine) 3302.13, 3151.23; $\mathrm{C}=\mathrm{O} 1600 ; \mathrm{C}=\mathrm{N}$ 1431.18; $\quad \mathrm{N}-\mathrm{H}$ 2920.23; C-N 1303.88 C-S 653.87; C-H (Aromatic) 
2852.72; -Cl 758.02. 1H NMR (400MHZ DMSO) $\delta$ ppm 8.0159 (aromatic), 7.5579 (aromatic), 8.7491 (sec. amide), $2.8912 \quad$ (-CH2), 4.2326 (aromatic C-NH).

2-((5H-1,2,4-triazol-3-yl)amino)-N-(5-(4chlorophenyl)-1,3,4-thiadiazol-2-yl)acetamide (D-IV). Mol.Formula- $\mathrm{C}_{8} \mathrm{H}_{6} \mathrm{IN}_{3} \mathrm{~S}$ Mol.wt- 335.77 M.P.-232$236^{\circ} \mathrm{C}$ Yield \%- 76; IR ( $\left.\mathrm{KBr}\right) \mathrm{cm}^{-1}-\mathrm{NH} 2$ (primary amine) 3302.13, 3149.76; $\mathrm{C}=\mathrm{O} 1590 ; \mathrm{C}=\mathrm{N} \quad 1490.97 ; \quad \mathrm{N}-\mathrm{H}$ 2920.23; C-N 1303.88; C-S 620; C-H(Aromatic) 2852.72; $-\mathrm{Cl} 756.10$.

\section{Anticonvulsant activity study}

Table 5: Anticonvulsant effect of some synthesized N-(5-(Substituted)-1,3,4-thiadiazol-2-yl)-2-((5-(substitutes)4H-1,2,4-triazol-3-yl)amino)acetamide, in mice using PTZ induced convulsions method

\begin{tabular}{|l|c|l|c|c|}
\hline \multicolumn{1}{|c|}{ Code } & $\begin{array}{c}\text { Dose } \\
\text { (mg/Kg, i.p.) }\end{array}$ & $\begin{array}{c}\text { Latency to induce } \\
\text { convulsions(Min) }\end{array}$ & $\begin{array}{c}\text { No. of } \\
\text { convulsions }\end{array}$ & \% Protection \\
\hline PTZ (Control) & 80 & $1.262 \pm 0.03412$ & 6 & 0 \\
\hline Diazepam & 2 & - & 0 & 100 \\
\hline \multirow{2}{*}{ A-II } & 10 & $6.804 \pm 1.294^{\text {NS }}$ & 3 & 40 \\
\cline { 2 - 5 } & 20 & $24.61 \pm 5.390^{* *}$ & 1 & 80 \\
\hline \multirow{2}{*}{ B-II } & 10 & $14.722 \pm 6.253^{*}$ & 2 & 60 \\
\hline \multirow{2}{*}{ C-II } & 20 & $28.072 \pm 1.928^{* *}$ & 2 & 60 \\
\hline \multirow{2}{*}{ D-III } & 10 & $12.028 \pm 0.4715^{\text {NS }}$ & 2 & 60 \\
\cline { 2 - 5 } & 20 & $27.04 \pm 2.960^{* *}$ & 1 & 80 \\
\cline { 2 - 5 } & 10 & $16.852 \pm 5.744 * *$ & 1 & 80 \\
\hline
\end{tabular}

$\mathrm{N}=5$, in each group; *: $\mathrm{P}<0.05 ; * *: \mathrm{P}<0.01$; NS: Non significant; One Way ANOVA followed by Dunnett's test. Values expressed as Mean \pm SEM

$\mathrm{X}(10 \mathrm{mg} / \mathrm{kg}$ dose $)$
Acute toxicity study: Acute toxicity studies was done for determining $\mathrm{LD}_{50}$. The $\mathrm{LD}_{50}$ was found to be $98.11 \mathrm{mg} / \mathrm{kg}$ for the synthesized N-(5-(Substituted)-1,3,4-thiadiazol-2yl)-2-((5-(substitutes)-4H-1,2,4-triazol-3-

yl)amino)acetamide derivatives. Two doses were selected for the anticonvulsant evaluation of compounds, dose I $10 \mathrm{mg} / \mathrm{kg}\left(1 / 10^{\text {th }}\right.$ that of $\left.\mathrm{LD}_{50}\right)$ and dose II $20 \mathrm{mg} / \mathrm{kg}$ (twice of dose I).

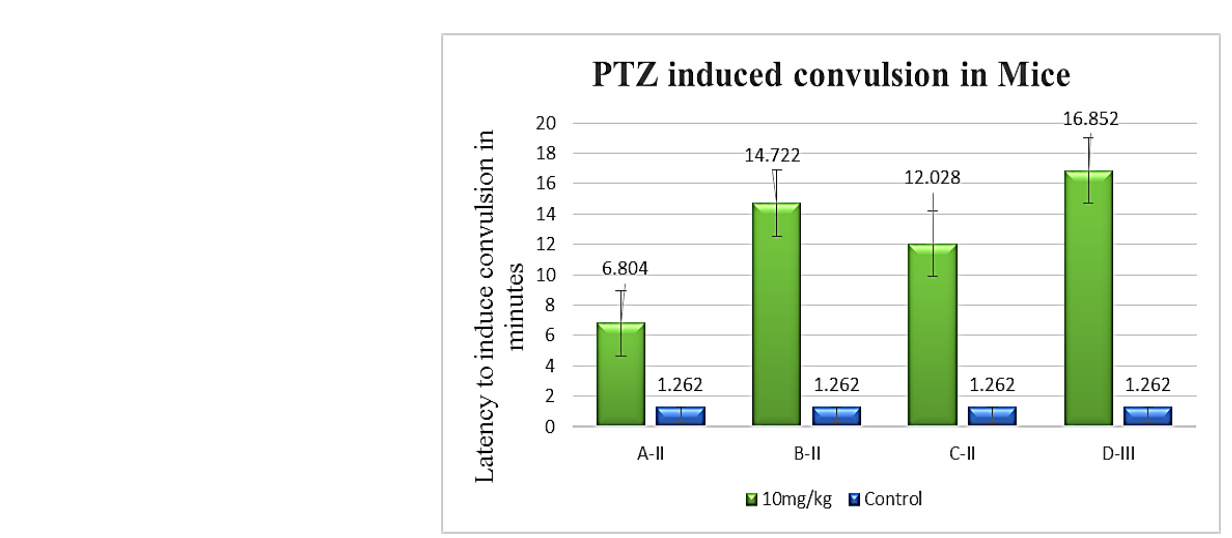




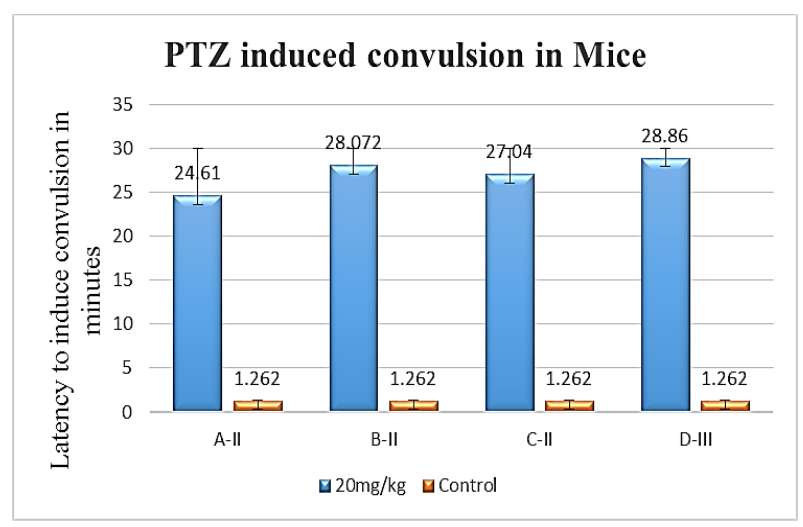

$\mathrm{Y}(20 \mathrm{mg} / \mathrm{kg}$ dose $)$

Fig. 3: Graphical representation of Latency to induce convulsions of some synthesized derivatives of $\mathrm{N}$-(5(Substituted)-1,3,4-thiadiazol-2-yl)-2-((5-(substitutes)-4H-1,2,4-triazol-3-yl)amino)acetamide in mice using PTZ induced convulsions

\section{Discussion}

In the present investigation, the 2-amino, 5substituted phenyl, 1,3,4-thiadiazole were coupled to 3amino 5-substituted phenyl 1,2,4-triazole with the aim of achieving anticonvulsant effect. The synthesized compounds were confirmed on the basis of IR, 1H-NMR. The analysis of structural features revealed that substitution of chloro group (either chloro group is present on thiadiazole or triazole ring) enhanced the anticonvulsant potential of the synthesized compounds. Acute toxicity studies was done for determining $\mathrm{LD}_{50}$. The $\mathrm{LD}_{50}$ was found to be $98.11 \mathrm{mg} / \mathrm{kg}$ for the synthesized $\mathrm{N}$ (5-(Substituted)-1,3,4-thiadiazol-2-yl)-2-((5-

(substitutes)-4H-1,2,4-triazol-3-yl) amino) acetamide derivatives. Two doses were selected for the anticonvulsant evaluation of compounds, dose I $10 \mathrm{mg} / \mathrm{kg}$ $\left(1 / 10^{\text {th }}\right.$ that of $\left.\mathrm{LD}_{50}\right)$ and dose II $20 \mathrm{mg} / \mathrm{kg}$ (twice of dose I). Some of the synthesized compounds were evaluated for their anticonvulsant effect. The pharmacological evaluation of the compounds showed increase in latency (onset time) to induce convulsions; decrease in the number of convulsions and increase in percentage of protection. The compounds D-III and B-II showed highest percentage of protection $(80 \%)$ at the dose of $20 \mathrm{mg} / \mathrm{kg}$ among the evaluated compounds compared to control.

\section{References}

1. A.P.G. Nikalje, M. Ghodke and A. Girbane. "GABA Modulating Agents: A Brief Review". Asian J Biol Sci 2011;4 (3);201-20.

2. Yasmin Khatoona*, Mohammad Shaquiquzzamanb, Vijender Singha, Mohammad Sarafrozc. "Synthesis, Characterization and Anticonvulsant Activity of Some Novel 4, 5-Disubstituted-1, 2, 4-Triazole Derivatives". J Applied Pharm Sci 2017;7(7):158-67.

3. Lincy Joseph, Mathew George, Prabha Mathews*. "A Review on Various Biological Activities of 1,3,4Thiadiazole Derivatives". Journal of Pharmaceutical, Chem Biol Sci 2015;3(3):329-45.

4. Ahmad S. Shawali. "1,3,4-Thiadiazoles of pharmacological interest: Recent trends in their synthesis via tandem 1,3dipolar cycloaddition: Review". J Adv Res (2014):1-17.
5. Ibrahim A. Flifel. "Preparation, Spectra characterization of new 1,2,4- Triazole Derivatives and its complexities with some transition metal ions". Int J Applied Engr Res (2017);24:14878-81.

6. Mukhtyar S. Saini. "Synthesis and biological significances of 1, 2, 4-triazole and its derivatives: a review". Int J Pharm Sci Res 2013;4(8):2866-79.

7. Parminder Kaur. "1,2,4-triazole: a review of pharmacological activities". Int Res J Pharm 2017;10-29.

8. Moayed S. AL-Gwady. "Synthesis of 2-Amino-5Substituted-1,3,4-Thiadiazoles (ATDA) and Their Derivatives Using Conventional and Microwave Techniques". J Raf Sci 2009;20(1):1- 7.

9. Georg Sjostedt, Leopold Gringas. "3-amino-1h-1,2,4triazole”. Organic Syntheses, Coll. 1955;3, 1946;26.

10. Kulkarni, S.K. (2009) "Handbook of Experimental Pharmacology”. (3rded. pp. 131-134). New Delhi: Vallabh prakashan.

11. Dyer, J.R. (2007) "Applications of absorption spectroscopy of organic compounds". 33-8. New Delhi: Prentice Hall of India. Pvt. Ltd. 